Samuel Kaplan, Department of Mathematics, University of North Carolina, Chapel Hill, NC 27599

\title{
ON UNBOUNDED ORDER CONVERGENCE
}

\begin{abstract}
Order convergence in Riesz spaces is defined only for order bounded sets; so does not apply, for example, to pointwise convergence in function spaces. There exists a generalization to not necessarily order bounced nets that includes the above pointwise convergence. In practice, application of this generalization is rather involved. We show that in the case of a Riesz space with a weak order unit, the definition takes a quite simple form, readily applicable to proofs.
\end{abstract}

A central tool in the study of Riesz spaces is order convergence. This is defined only for order bounded sets, so cannot be applied to situations in which the nets concerned are not necessarily order bounded. For example, it does not include pointwise convergence of nets in a Riesz space of functions on a set $X$.

There exists a generalization of order convergence which applies to all nets in a Riesz space and includes, among others, the above pointwise convergence (and reduces, of course, to order convergence for order bounded nets). To my knowledge, it was first defined and applied by H. Nakano ([4], [5]) (under the name "individual convergence" in the second reference). Following DeMarr [1], we will call it "unbounded order convergence".

The various definitions of unbounded order convergence do not lend themselves easily to proofs of theorems. However, in our work on the Lebesgue integral in the bidual of $C(X)$ for $X$ compact (cf. [3]), and in a planned sequel on measurability in a superspace of the bidual, we deal with spaces Dedekind complete spaces with a weak order unit - in which unbounded order convergence has a simple characterization. We present the details in the present note.

\footnotetext{
Mathematical Reviews subject classification: 46A110, 06F20

Received by the editors January 3, 1997

*We are indebted to the referee, under whose suggestions the paper has been considerably shortened and extraneous material eliminated from propositions.
} 


\section{Notation}

$\mathbb{N}$ will denote the natural numbers, $\mathbb{R}$ the reals, and $\mathbb{R}_{+}$the non-negative reals. $E$ will be a fixed Riesz space. $a, b, c, d, e$ will denote elements of $E$, and $\lambda, \kappa$, $\rho$ elements of $\mathbb{R}$. For notation and terminology not specially defined, we refer the reader to [2] or any book on Riesz spaces.

Subsets of $E$ will be denoted by $A$ or $B$ or by $\left\{a_{\alpha}\right\}$, it being understood that the subscript runs through an index set $\mathcal{A}$. However, if we speak of a net $\left\{a_{\alpha}\right\}$, we will mean that $\mathcal{A}$ is a directed set, that is, it is endowed wit an order $\leq$ such that for each pair $\alpha_{1}, \alpha_{2}$, there exists $\alpha_{3} \geq \alpha_{1}, \alpha_{2}$.

If an (order bounded) set $\left\{a_{\alpha}\right\}$ in $E$ order converges to $a \in E$, we will write

$$
a_{\alpha} \longrightarrow a .
$$

If $E$ is Dedekind complete, order convergence can be described in terms of $\lim \sup _{a} a_{\alpha}$ and $\liminf _{\alpha} a_{\alpha}$. Specifically

$$
\begin{aligned}
& \operatorname{lim\operatorname {sup}a_{\alpha }}=\wedge_{\alpha}\left(\vee_{\beta \geq \alpha} a_{\beta}\right), \\
& \liminf _{\alpha} a_{\alpha}=\vee_{\alpha}\left(\wedge_{\beta \geq \alpha} a_{\beta}\right) ;
\end{aligned}
$$

and the statement $a_{\alpha} \longrightarrow a$ is equivalent to

$$
\liminf _{\alpha} a_{\alpha}=a=\limsup _{\alpha} a_{\alpha} .
$$

Given $A \subset E$, the disjoint $A^{d}$ of $A$ is the set $\{b \in E:|b| \wedge|a|=0$ for all $a \in A\}$. $A^{d}$ is always a band. Suppose $E$ is Dedekind complete. Then (by the classical Riesz theorem) every band $H$ of $E$ is a projection band: $E=H \oplus H^{d}$. It follows that for every $b \in E$, we can talk about the component $b_{H}$ of $b$ in $H$. For $B \subset E$, we denote the set $\left\{b_{H}: b \in B\right\}$ by $B_{H}$. Finally, if $H$ is the band generated by a single $a \in E$, we will feel free to write $b_{a}$ for $b_{H}$ and $B_{a}$ for $B_{H}$.

\section{Unbounded Order Convergence}

We will use the following lemma for which we refer the reader to $\S \S 1,2$ in [2].

1.1. For $\lambda \in \mathbb{R}_{+}$and $a_{0} \in E$, the following operations on $E$ preserve suprema and infima:

$$
a \longrightarrow \lambda a, a \longrightarrow a+a_{0}, a \longrightarrow a \vee a_{0}, \text { and } a \longrightarrow a \wedge a_{0} .
$$

It follows that they also preserve limsup and liminf, so, a fortiori, order convergence. 
A net $\left\{a_{\alpha}\right\}$ (not necessarily order bounded) in $E$ will be said to converge to $a \in E$ unboundedly if for every pair $b, c \in E$ with $b \leq c$,

$$
\left(a_{\alpha} \wedge c\right) \vee b \longrightarrow(a \wedge c) \vee b
$$

And we will write

$$
a_{\alpha} \stackrel{u}{\rightarrow} a
$$

\section{Remarks.}

(1) The above convergence can also be written $\left(a_{\alpha} \vee b\right) \wedge c \longrightarrow(a \vee b) \wedge c$.

(2) In the above definition, it suffices that the convergence hold for pairs $b, c$ such that $b \leq 0 \leq c$.

(3) For an order bounded net, unbounded order convergence clearly reduces to order convergence.

Some elementary properties:

1.2. (1) If $a_{\alpha} \stackrel{u}{\longrightarrow} a$, then $\rho a_{\alpha} \stackrel{u}{\rightarrow} \rho a$ for all $\rho \in \mathbb{R}$. In particular $-a_{\alpha} \stackrel{u}{\longrightarrow}-a$.

(2) If $a_{\alpha} \stackrel{u}{\longrightarrow} a$ and $d_{\alpha} \stackrel{u}{\longrightarrow} d$, then

$$
\begin{aligned}
& a_{\alpha} \vee d_{\alpha} \stackrel{u}{\rightarrow} a \vee d, \\
& a_{\alpha} \wedge d_{\alpha} \stackrel{u}{\rightarrow} a \wedge d .
\end{aligned}
$$

One consequence: If $a_{\alpha} \leq d_{\alpha}$ for all $\alpha$, then $a \leq d$.

(3) Under unbounded order convergence, limits are unique: If $a_{\alpha} \stackrel{u}{\rightarrow} a$ and $a_{\alpha} \stackrel{u}{\rightarrow} a^{\prime}$, then $a=a^{\prime}$.

(4) If $a_{\alpha} \stackrel{u}{\longrightarrow} a, d_{\alpha} \stackrel{u}{\longrightarrow} a$, and $a_{\alpha} \leq e_{\alpha} \leq d_{\alpha}$ for all $\alpha$, then $e_{\alpha} \stackrel{u}{\rightarrow} a$.

The verifications are straightforward.

Contained in the above are the following two simple properties. we state them formally for reference below.

1.3. For a net $\left\{a_{\alpha}\right\}$ in $E$ and $a \in E$, the following are equivalent:

$1^{\circ} a_{\alpha} \stackrel{u}{\longrightarrow} 0$,

$2^{\circ}\left|a_{\alpha}\right| \stackrel{u}{\longrightarrow} 0$.

The proof of the following is due to the referee. It replaces the previous one in the original draft. 
1.4. If $a_{\alpha} \stackrel{u}{\longrightarrow} a$ and $d_{\alpha} \stackrel{u}{\longrightarrow} d$, then

$$
a_{\alpha}+d_{\alpha} \stackrel{u}{\longrightarrow} a+d .
$$

Proof. For each $\alpha$,

$$
\begin{aligned}
0 & \leq\left|\left(a_{\alpha}+d_{\alpha}\right)-(a+d)\right| \\
& \leq\left|a_{\alpha}-a\right|+\left|d_{\alpha}-d\right| \\
& \leq 2\left|a_{\alpha}-a\right| \vee 2\left|d_{\alpha}-d\right| .
\end{aligned}
$$

By $1.3,1.4$, and (2) in 1.2, this last converges to 0 unboundedly. Hence by (4) in 1.2 ,

$$
\left|\left(a_{\alpha}+d_{\alpha}\right)-(a+d)\right| \stackrel{u}{\longrightarrow} 0 .
$$

Applying 1.4 and 1.3 again gives us the desired result.

1.5. For a net $\left\{a_{\alpha}\right\}$ in $E$ and $a \in E$, the following are equivalent:

$1^{\circ} a_{\alpha} \stackrel{u}{\longrightarrow} a$;

$2^{\circ} \quad$ (i) $\left(a_{\alpha}\right)^{+} \stackrel{u}{\rightarrow} a^{+}$,

(ii) $\left(a_{\alpha}\right)^{-} \stackrel{u}{\longrightarrow} a^{-}$.

And they imply

$$
\left|a_{\alpha}\right| \stackrel{u}{\longrightarrow}|a| .
$$

Proof. Assume $1^{\circ}$. Then by 1.3 and $1.4,\left|a_{\alpha}-a\right| \stackrel{u}{\longrightarrow} 0$. Now

$$
0 \leq\left|\left(a_{\alpha}\right)^{+}-a^{+}\right| \leq\left|a_{\alpha}-a\right| \text { for all } \alpha, \text { so }\left|\left(a_{\alpha}\right)^{+}-a^{+}\right| \stackrel{u}{\longrightarrow} 0
$$

((4) in 1.2), so $a_{\alpha}^{+} \stackrel{u}{\longrightarrow} a^{+}$. Since $-a_{\alpha} \stackrel{u}{\longrightarrow}-a$, the same argument gives that $\left(a_{\alpha}\right)^{-} \stackrel{u}{\longrightarrow} a^{-}$. That $2^{\circ}$ implies $1^{\circ}$ follows from 1.5 above. And the final statement follows from ||$a_{\alpha}|-| a|| \leq\left|a_{\alpha}-a\right|$ and (4) in 1.2.

Remark. If in the above, $a=0$, then the final statement is equivalent to the first two.

We also record

1.6. If $E$ is the direct sum of two bands: $E=H_{1} \oplus H_{2}$, the following are equivalent:

$1^{\circ} a_{\alpha} \stackrel{u}{\longrightarrow} a ;$

$2^{\circ} \quad$ (i) $\quad\left(a_{\alpha}\right)_{H_{1}} \stackrel{u}{\longrightarrow} a_{H_{1}}$,

(ii) $\quad\left(a_{\alpha}\right)_{H_{2}} \stackrel{u}{\longrightarrow} a_{H_{2}}$. 


\section{Weak Order Unit}

In the present $\S$ we assume $E$ contains a weak order unit, which we denote by $\mathbf{1}$ (for all $a \in E, a \wedge \mathbf{1}=0$ implies $a=0$ ). Also that $E$ is Dedekind complete. This last assumption is for simplicity. Some of the following propositions hold under weaker assumptions, but the spaces in which we are interested are Dedekind complete.

2.1. For a net $\left\{a_{\alpha}\right\}$ in $E_{+}$, the following are equivalent:

$1^{\circ} a_{\alpha} \stackrel{u}{\longrightarrow} 0$,

$2^{\circ} a_{\alpha} \wedge \mathbf{1} \longrightarrow 0$.

Proof. Assume $2^{\circ}$ holds. To establish $1^{\circ}$, it suffices to show that for every $c \in E_{+}, a_{\alpha} \wedge c \longrightarrow 0$. We show the equivalent:

$$
\text { (i) } \quad \limsup _{\alpha}\left(a_{\alpha} \wedge c\right)=0
$$

(Since $E$ is Dedekind complete, the left side exists.)

$$
\begin{aligned}
& 0 \leq\left[\lim \sup \left(a_{\alpha} \wedge c\right)\right] \wedge \mathbf{1} \\
& =\limsup _{\alpha}\left(a_{\alpha} \wedge c \wedge \mathbf{1}\right) \\
& \leq \lim \sup \left(a_{\alpha} \wedge \mathbf{1}\right) \\
& =0 \text {. }
\end{aligned}
$$

Thus $\left[\lim \sup _{\alpha}\left(a_{\alpha} \wedge c\right)\right] \wedge \mathbf{1}=0$. Since $\mathbf{1}$ is a weak order unit, we have (i).

Combining 1.3, 1.4, and the above gives us:

2.2 Theorem. For a net $\left\{a_{\alpha}\right\}$ in $E$ and $a \in E$, the following are equivalent:

$1^{\circ} a_{\alpha} \stackrel{u}{\longrightarrow} a$,

$2^{\circ}\left|a_{\alpha}-a\right| \wedge \mathbf{1} \longrightarrow 0$.

We establish a second characterization of unbounded order convergence in $E$ (2.8 below). The reader will notice its resemblance to convergence in measure.

Recall that for each $a \in E, \mathbf{1}_{\alpha}$ denotes the component of $\mathbf{1}$ in the band generated by $a$. So, for each $a \in E$ and $\lambda \in \mathbb{R}, \mathbf{1}_{(a-\lambda \mathbf{1})^{+}}$is the component of 
$\mathbf{1}$ in the band generated by $(a-\lambda \mathbf{1})^{+}$. If $E=\mathbb{R}^{X}$, the space of real functions on a set $X$ (or more generally, a $\sigma$-order closed subset of $\mathbb{R}^{X}$ ), and $\mathbf{1}$ is the constant function: $\mathbf{1}(x)=1$ for all $x$, then for $f \in E$ and $\lambda \in \mathbb{R}, \mathbf{1}_{(f-\lambda \mathbf{1})^{+}}$is the characteristic function of the set $\{x \in X: f(x)>\lambda\}$. The components of $\mathbf{1}$ of the form $\mathbf{1}_{(a-\lambda \mathbf{1})}+$ are thus generalizations of such characteristic functions. This explains their role in the paper.

We record two properties which we will need. The first is an alternate form of the statement that $\mathbf{1}$ is a weak order unit.

2.3. For $a \in E$, the following are equivalent:

$1^{\circ} \mathbf{1}_{\alpha}=0$,

$2^{\circ} a=0$.

2.4. Given $a \in E_{+}$, then for $\lambda>0$,

$$
\mathbf{1}_{(a-\lambda \mathbf{1})+} \leq \frac{1}{\lambda} a
$$

Cf. (17.9) in [2] for a proof.

We proceed to develop our characterization.

2.5. Given a set $\left\{a_{\alpha}\right\}$ in $E_{+}$,

$$
\wedge_{\alpha} \mathbf{1}_{a_{\alpha}}=0 \text { implies } \quad \wedge_{\alpha} a_{\alpha}=0 .
$$

The converse is false.

Proof. For every $\alpha, 0 \leq a_{\alpha} \wedge \mathbf{1} \leq \mathbf{1}_{a_{\alpha}}$, hence

$$
0 \leq\left(\wedge_{\alpha} a_{\alpha}\right) \wedge \mathbf{1}=\wedge_{\alpha}\left(a_{\alpha} \wedge \mathbf{1}\right) \leq \wedge_{\alpha} \mathbf{1}_{a_{\alpha}}=0 .
$$

Thus $\left(\wedge_{\alpha} a_{\alpha}\right) \wedge \mathbf{1}=0$, whence $\wedge_{\alpha} a_{\alpha}=0$. To see that the converse is false, set $a_{n}=\frac{1}{n} \mathbf{1}(n=1,2, \ldots)$.

However

2.6. For a set $\left\{a_{\alpha}\right\}$ in $E_{+}$, the following are equivalent:

$1^{\circ}$ For every $\lambda>0, \wedge_{\alpha} \mathbf{1}_{\left(a_{\alpha}-\lambda \mathbf{1}\right)^{+}}=0$,

$2^{\circ} \wedge_{\alpha} a_{\alpha}=0$.

Proof. Assume $1^{\circ}$. We show $\wedge_{\alpha} a_{\alpha} \leq \lambda \mathbf{1}$ for every $\lambda>0$. Fix $\lambda$. By 2.5, $\lambda_{\alpha}\left(a_{\alpha}-\lambda \mathbf{1}\right)^{+}=0$. Hence $\wedge_{\alpha}\left(a_{\alpha}-\lambda \mathbf{1}\right) \leq 0$, which can be written $\wedge_{\alpha} a_{\alpha} \leq \lambda \mathbf{1}$. The converse implication follows from 2.4. 
Remark. $1^{\circ}$ is clearly equivalent to:

$$
\left(1^{\circ}\right)^{\prime} \quad \wedge_{\alpha} \mathbf{1}_{\left(a_{\alpha}-n \mathbf{1}\right)}=0 \quad(n=1,2, \ldots) .
$$

2.7. Given a net $\left\{a_{\alpha}\right\}$ in $E_{+}$,

$$
\mathbf{1}_{a_{\alpha}} \longrightarrow 0 \quad \text { implies } \quad a_{\alpha} \stackrel{u}{\longrightarrow} 0 .
$$

The converse is false.

The proof is essentially the same as for 2.5, using 2.1 .

However, again,

2.8 Theorem. For a net $\left\{a_{\alpha}\right\}$ in $E_{+}$, the following are equivalent:

$1^{\circ}$ For every $\lambda>0, \mathbf{1}_{\left(a_{\alpha}-n \mathbf{1}\right)} \longrightarrow 0$,

$2^{\circ} a_{\alpha} \stackrel{u}{\longrightarrow} 0$.

Proof. Assume $1^{\circ}$, and suppose first that $\left\{a_{\alpha}\right\}$ is order bounded. We show that

$$
\limsup a_{\alpha} \leq \lambda \mathbf{1} \text { for every } \lambda>0 \text {. }
$$

$\alpha$

Fix $\lambda$. By 2.7, $\left(a_{\alpha}-\lambda \mathbf{1}\right)^{+} \stackrel{u}{\longrightarrow} 0$. In particular, $\lim \sup _{\alpha}\left(a_{\alpha}-\lambda \mathbf{1}\right)^{+}=0$, hence $\lim \sup _{\alpha}\left(a_{\alpha}-\lambda \mathbf{1}\right) \leq 0$, which can be written $\lim \sup _{\alpha} a_{\alpha} \leq \lambda \mathbf{1}$.

Now drop the supposition that $\left\{a_{\alpha}\right\}$ is order bounded. We show $a_{\alpha} \wedge \mathbf{1} \rightarrow 0$ (hence, by $2.1, a_{\alpha} \stackrel{u}{\longrightarrow} 0$ ). For every $\lambda>0$,

$$
0 \leq \mathbf{1}_{\left(a_{\alpha} \wedge \mathbf{1}-\lambda \mathbf{1}\right)^{+}} \leq \mathbf{1}_{\left(a_{\alpha}-\lambda \mathbf{1}\right)^{+}} \longrightarrow 0
$$

Since $\left\{a_{\alpha} \wedge \mathbf{1}\right\}$ is order bounded, it follows from the first part of the proof that $a_{\alpha} \wedge \mathbf{1} \longrightarrow 0$. As before, the converse implication follows from 2.4.

Remark. Again, $1^{\circ}$ is clearly equivalent to:

$$
\left(1^{\circ}\right)^{\prime} \quad \mathbf{1}_{\left(a_{\alpha}-n \mathbf{1}^{+}\right)} \longrightarrow 0 \quad(n=1,2, \ldots)
$$

\section{A Useful Theorem}

A Riesz space $E$ is called laterly complete if for every set $\left\{a_{\alpha}\right\}$ in $E$ satisfying

$$
a_{\alpha_{1}} \wedge a_{\alpha_{2}}=0 \text { for } \alpha_{1} \neq \alpha_{2}
$$


$\wedge_{\alpha} a_{\alpha}$ exists. We will say it is laterally $\sigma$-complete if this property holds for countable sets $\left\{a_{n}\right\}$. For a set $X, \mathbb{R}^{X}$ is an example of a laterly complete Riesz space.

In the present $\S$, we confine ourselves to $E$ Dedekind $\sigma$-complete and to sequences. Note that the results of $\S \S 1,2$ hold for sequences when the space $E$ is Dedekind $\sigma$-complete.

Nakano states in [5] that if $E$ is Dedekind $\sigma$-complete and laterally $\sigma$ complete, then every sequence which order converges unboundedly is order bounded - hence, as we have noted, order converges. We present a proof of this theorem. Our original proof required that $E$ have a weak order unit. The referee supplied the following Lemma, enabling us to eliminate that condition.

3.1 Lemma. Given that $E$ is Dedekind $\sigma$-complete and laterally $\sigma$-complete. Then for a countable subset $\left\{a_{n}\right\}$ of $E$, the band $H$ generated by $\left\{a_{n}\right\}$ has the following properties:

(1) $H$ is a projection band;

(2) $H$ is Dedekind $\sigma$-complete;

(3) $H$ is laterally $\sigma$-complete;

(4) H has a weak order unit.

Proof. As is well known, (1) follows from the Dedekind $\sigma$-completeness of $E$; and (2) and (3) are straightforward. We prove (4).

With no loss of generality, we can assume $\left\{a_{n}\right\} \subset E_{+}$. For each $n=$ $1,2, \ldots$, let $G_{n}$ be the band of $E$ generated by $\left\{a_{1}, \ldots, a_{n}\right\}$. Then (as for (1)) the $G_{n}$ 's are projection bands, and, denoting order closure by "cl", $H=$ $\operatorname{cl}\left(\cup_{n} G_{n}\right)$. Since $\cup_{n} G_{n}=\sum_{n} G_{n}$, this can also be written $H=\operatorname{cl}\left(\sum_{n} G_{n}\right)$.

Now set $H_{1}=G_{1}$, and for each $n>1$, set $H_{n}=G_{n} \cap\left(G_{n-1}\right)^{d}$. Then

The $H_{n}$ 's are projection bands;

$$
\begin{aligned}
& H_{n} \cap H_{m}=0 \text { for } n \neq m ; \\
& H=\operatorname{cl}\left(\sum_{n} H_{n}\right) .
\end{aligned}
$$

Finally, set $b_{n}=\left(a_{n}\right)_{H_{n}}(n=1,2, \ldots)$. Then

(i) $b_{n} \wedge b_{m}=0 \quad n \neq m$;

(ii) for each $n, b_{n}$ is a weak order unit for $H_{n}$. 
(i) is clear; we show (ii). $G_{n}$ is the band generated by $a_{1} \vee \ldots \vee a_{n}$, which is therefore a weak order unit for $G_{n}$. It follows $\left(a_{1} \vee \ldots \vee a_{n}\right)_{H_{n}}=b_{n}$.

Since $H$ is laterally $\sigma$-complete, (i) gives us that $b=\vee_{n} b_{n}$ exists in $H$, and it follows easily from (ii) that it is a weak order unit for $H$.

3.2 (Nakano). If $E$ is Dedekind $\sigma$-complete and laterally $\sigma$-complete, then every sequence $\left\{a_{n}\right\}$ in $E$ which order converges unboundedly is order bounded.

Proof. With no loss of generality, we can assume that $\left\{a_{n}\right\} \subset E_{+}$and $a_{n} \stackrel{u}{\longrightarrow}$ 0 . From the Lemma, we can confine ourselves to the band of $E$ generated by $\left\{a_{n}\right\}$, so for simplicity, we will simply assume that $E$ contains a weak order unit 1.

By $2.8, \mathbf{1}_{\left(a_{n}-\mathbf{1}\right)}+\longrightarrow 0$. For each $n$, set

$$
\begin{aligned}
& d_{n}=\wedge_{m \geq n} \mathbf{1}_{\left(a_{m}-\mathbf{1}\right)^{+}}, \\
& F_{n}=\text { the band generated by } d_{n}, \\
& c_{n}=1-d_{n} .
\end{aligned}
$$

Then

$d_{n} \downarrow 0 ;$

$F_{1} \supset F_{2} \supset \ldots$, with $\cap_{n} F_{n}=0$;

$c_{n} \uparrow \mathbf{1}$

$\left(F_{1}\right)^{d} \subset\left(F_{2}\right)^{d} \subset \ldots$, with $E=\operatorname{cl}\left[\sum_{n}\left(F_{n}\right)^{d}\right] ;$

for each $n$,

$$
\left(a_{m}\right)_{\left(F_{n}\right)^{d}} \leq \mathbf{1} \text { for all } m \geq n .
$$

Now set

$$
\begin{aligned}
& e_{1}=c_{1}, e_{n}=c_{n}-c_{n-1} \text { for } n>1 ; \\
& H_{n}=\text { the band generated by } e_{n}(n=1,2, \ldots) .
\end{aligned}
$$

Then

(i) $H_{n} \cap H_{m}=0 \quad n \neq m$;

(ii) $\sum_{1}^{n} H_{i}=\left(F_{n}\right)^{d}(n=1,2, \ldots)$;

(iii) $E=\operatorname{cl}\left(\sum_{n} H_{n}\right)$; 
(iv) for each $n$,

$$
\left(a_{m}\right)_{H_{n}} \leq e_{n} \text { for } m \geq n .
$$

It follows from (iv) that for each $n$,

$$
\left\{\left(a_{m}\right)_{H_{n}}: m=1,2, \ldots\right\} \leq\left(\wedge_{m=1}^{n}\left(a_{m}\right)_{H_{n}}\right) \wedge e_{n} .
$$

Denote the right side by $b_{n}$. The $b_{n}$ 's are mutually disjoint, hence, since $E$ is laterally $\sigma$-complete, $b=\vee_{n} b_{n}$ exists. Then $\left\{a_{n}\right\} \leq b$, and we are through.

3.3 Corollary. If $E$ is Dedekind $\sigma$-complete and laterally $\sigma$-complete, then for a sequence $\left\{a_{n}\right\}$ in $E$ and $a \in E$, the following are equivalent:

$1^{\circ} a_{n} \stackrel{u}{\longrightarrow} a$,

$2^{\circ} a_{n} \longrightarrow a$.

\section{References}

[1] R. DeMarr, Partially order linear spaces and locally convex linear topological spaces, Ill. J. Math. 8 (1964), 601-606.

[2] S. Kaplan, The Bidual of $C(X)$, North Holland, 1985.

[3] S. Kaplan, Lebesgue theory in the Bidual of $C(X)$, Memoirs A.M.S. 579 (1996).

[4] H. Nakano, Teilweise geordnete Algebra, Japanese J. Math 17 (1941), $425-511$.

[5] H. Nakano, Ergodic theorems in semi-ordered linear spaces, Ann. Math. 49 (1948), 538-556. 